\title{
PIXLEY-ROY AND THE SOUSLIN LINE
}

\section{MARY ELLEN RUDIN}

\begin{abstract}
Necessary and sufficient conditions are given for normality and metricity of the Pixley-Roy space over a subset of the Souslin line.
\end{abstract}

The purpose of this paper is to answer a question of E. Parker: for which subsets $X$ of a Souslin [1] line $S$ is the Pixley-Roy [2] space $P_{X} R$ over $X$ normal? for which is it metric?

Without loss of generality, we assume that $S$ is compact, connected, and without nontrivial separable subintervals. Then: $S=\cup_{\alpha \in \omega_{1}} K_{\alpha}$ where each $K_{\alpha}$ is a Cantor set and $K_{\alpha} \subset K_{\beta}$ for all $\alpha<\beta$. Let

$$
D_{\alpha}=\left(X-\bigcup_{\beta<\alpha} K_{\beta}\right) \cap \mathrm{cl}\left(\bigcup_{\beta<\alpha} K_{\beta} \cap X\right) .
$$

Consider statements:

(A) $\left\{\alpha \in \omega_{1} \mid D_{\alpha} \neq \varnothing\right\}$ is not stationary in $\omega_{1}$.

(B) $P_{\left(X \cap K_{\alpha}\right)} R$ is metric for all $\alpha \in \omega_{1}$.

(C) $P_{\left(X \cap K_{\alpha}\right)} R$ is normal for all $\alpha \in \omega_{1}$.

We prove:

(I) $P_{X} R$ is metric if and only if both (A) and (B) hold.

(II) $P_{X} R$ is normal if and only if both (A) and (C) hold.

If $W$ is a subset of a Cantor set $K$, we know the following:

(D) [2] $P_{W} R$ is metric if and only if $W$ is countable.

(E) [Theorem 4 of this paper] $P_{W} R$ is normal if and only if $W^{n}$ is a $Q$-set ${ }^{1}$ for all $n \in N$.

(F) [4] It is consistent with ZFC that both there exists a Souslin line and $P_{W} R$ is normal only if it is also metric.

(G) [3] It is consistent with ZFC that there exist both a Souslin line and a $W \subset K$ such that $P_{W} R$ is normal but not metric.

Using (D) and (E), (I) and (II) become

(I') $P_{X} R$ is metric if and only if (A) holds and $X \cap K_{\alpha}$ is countable for all $\alpha$.

(II') $P_{X} R$ is normal if and only if (A) holds and $\left(X \cap K_{\alpha}\right)^{n}$ is a $Q$-set for all $n \in N$ and $\alpha \in \omega_{1}$.

$P_{X} R$ is always a Moore space [2]; thus $P_{X} R$ is a normal nonmetrizable Moore space if and only if (A) and (C) hold but (B) does not. By (F) and (G)

Received by the editors June 28, 1977 and, in revised form, February 20, 1978.

AMS (MOS) subject classifications (1970). Primary 02K25, 54A25, 04A30, 54D20; Secondary 54B10, 54D45.

${ }^{1}$ A space $S$ is a $Q$-set provided every subset of $S$ is a $G_{8}$-set in $S$. 
it is independent of ZFC whether there is an $X$ such that $P_{X} R$ is a normal nonmetrizable Moore space even if one requires that $X-K_{\alpha} \neq \varnothing$ for all $\alpha \in \omega_{1}$.

My reasons for bothering with all of this are:

(1) I had expected $P_{X} R$ to be metric only if $X$ were countable (and $P_{X} R$ to be normal only if $X$ were contained in a Cantor set).

(2) I think the following problem is important and I do not know how to solve it. Suppose that $W$ is a $Q$-set (contained in a Cantor set). Is $W^{2}$ (or $W^{n}$ ) a $Q$-set? It is certainly consistent with ZFC that there exist a $Q$-set and that the answer be yes for all $Q$-sets $W$. I conjecture that it is also consistent that the answer be no.

In proving Theorems 2 and 3 we do not use the fact that $S$ has no uncountable family of disjoint open intervals; i.e. $S$ could be any linear space with the structure described in paragraph two; i.e. $S$ could be an Aronszajn line.

The Pixley-Roy space $P_{X} R$ over a space $X$ is the set of all finite subsets of $X$. If $F \in P_{X} R$ and $U$ is open in $X$ then $\left\{G \in P_{X} R \mid F \subset G \subset U\right\}$ is a basic open set in $P_{X} R$. Throughout the paper we assume that $X \subset S$ and $S, K_{\alpha}$, and $D_{\alpha}$ are as defined in the second paragraph. Conditions (B) and (C) are obviously necessary for (I) and (II) respectively; we begin by proving that (A) is necessary:

THEOREM 1. If $\left\{\alpha \in \omega_{1} \mid D_{\alpha} \neq \varnothing\right\}$ is stationary in $\omega_{1}$, then $P_{X} R$ is not normal.

Proof. Using $<$ here for the order in $S$, let

$$
L_{\alpha}=\left\{x \in D_{\alpha} \mid x \in \operatorname{cl}\left\{y \in X \cap\left(\bigcup_{\beta<\alpha} K_{\beta}\right) \mid y<x\right\}\right\}
$$

and

$$
R_{\alpha}=\left\{x \in D_{\alpha} \mid x \in \mathrm{cl}\left\{y \in X \cap\left(\bigcup_{\beta<\alpha} K_{\beta}\right) \mid y>x\right\}\right\} .
$$

Since $D_{\alpha}=L_{\alpha} \cup R_{\alpha}$, we assume without loss of generality that $\left\{\alpha \in \omega_{1} \mid L_{\alpha}\right.$ $\neq \varnothing\}$ is stationary in $\omega_{1}$.

Let $\mathcal{G}$ be the set of all nontrivial open subintervals of $S$. There is an $S_{0} \in \mathcal{G}$ such that, for all $I \in G$ with $I \subset S_{0},\left\{\alpha \in \omega_{1} \mid L_{\alpha} \cap I \neq \varnothing\right\}$ is stationary in $\omega_{1}$. To see this let $G^{*}$ be a maximal family of disjoint members of $\mathscr{G}$ such that for each $I \in g^{*}$ there is a closed unbounded subset $\Omega_{I}$ of $\omega_{1}$ with $L_{\alpha} \cap I=\varnothing$ for all $\alpha \in \Omega_{I}$. If there is an $S_{0} \in \mathscr{G}$ contained in $S-\cup\left(g^{*}\right)$, then $S_{0}$ clearly has the desired properties. Otherwise $\cup\left(G^{*}\right)$ is dense in $S$ and hence, since $G$ is countable, $S-\cup\left(g^{*}\right)$ is separable. So there is a $\beta$ with $\left(S-\cup\left(g^{*}\right)\right) \subset$ $K_{\beta}$. But then $\left\{\alpha \in \omega_{1} \mid L_{\alpha} \neq \varnothing\right\}$ is not stationary since it does not meet the closed unbounded set $\left\{\alpha>\beta \mid \alpha \in \cap_{I \in 9^{*}} \Omega_{I}\right\}$.

By induction, for each $\alpha \in \omega_{1}$ choose $\delta_{\alpha} \in \omega_{1}$ and $y_{\alpha} \in L_{\delta_{\alpha}} \cap S_{0}$ in such a 
way that $\delta_{\alpha}>\sup \left\{\Delta_{\beta} \mid \beta<\alpha\right\}$. Let $Y=\left\{y_{\alpha} \mid \alpha \in \omega_{1}\right\}, Z=X-Y$, and $Y^{*}$ and $Z^{*}$ be the set of all singletons from $Y$ and $Z$, respectively. Since $Y^{*}$ and $Z^{*}$ are closed and disjoint in $P_{X} R$, assuming that $P_{X} R$ is normal there are disjoint open sets $U$ and $V$ in $P_{X} R$ such that $Y^{*} \subset U$ and $Z^{*} \subset V$.

For each $\alpha \in \omega_{1}$, since $y_{\alpha} \in D_{\delta_{\alpha}}$ and $\delta_{\alpha}>\sup \left\{\delta_{\beta} \mid \beta<\alpha\right\}, y_{\alpha} \notin \operatorname{cl}\left\{y_{\beta} \mid \beta<\right.$ $\alpha$. Thus there is a $J_{\alpha} \in 9$ such that $y_{\alpha}$ is the left end point of $J_{\alpha}$ and $J_{\alpha} \cap\left\{y_{\beta} \mid \beta<\alpha\right\}=\varnothing$. Since $\left\{y_{\alpha}\right\} \in Y^{*} \subset U, J_{\alpha}$ can be chosen in such a way that the unordered pair $\left\{y_{\alpha}, x\right\} \in U$ for all $x \in\left(J_{\alpha} \cap X\right)$.

Using the same type of argument used in finding $S_{0}$, we can find an $S_{1} \subset S_{0}$ with $S_{1} \in \mathcal{G}$ such that if $I \subset S_{1}$ and $I \in \mathcal{G}$, then $I \cap Y \neq \varnothing$.

For each $\alpha \in \omega_{1}$ choose a maximal family $g_{\alpha}$ of disjoint members of $\left\{J_{\beta} \mid \beta>\alpha\right\}$. In $\omega_{1}$ choose $\alpha^{*}>\sup \left\{\delta_{\beta} \mid J_{\beta} \in \mathscr{I}_{\alpha}\right\}$. Observe that if $x \in S_{1} \cap$ $L_{\gamma}$ for some $\gamma>\alpha^{*}$, then there is a $J \in \mathscr{G}_{\alpha}$ with $x \in J$. To see this suppose the contrary. Since $x \in D_{\gamma}$ and $\gamma>\alpha^{*}$, there is an $I \in \mathcal{G}$ such that $I \subset S_{1}, x$ is the left end point of $I$, and $I \cap\left\{y_{\beta} \mid \beta \leqslant \alpha^{*}\right\}=\varnothing$. Since $I \subset S_{1}$, there is a $\rho \in \omega_{1}$ with $y_{\rho} \in I$. Suppose that $\beta<\alpha^{*}$. If $y_{\rho}<y_{\beta}$ in $S$, then $y_{\beta} \notin J_{\rho}$ by definition; thus $J_{\rho} \cap J_{\beta}=\varnothing$ since $y_{\beta}$ is the left end point of $J_{\beta}$. If $y_{\rho}>y_{\beta}$ in $S$, then, since $y_{\beta} \notin I, y_{\beta}<x$; hence, since $x \notin J_{\beta}, J_{\rho} \cap J_{\beta}=\varnothing$. Thus $J_{\rho} \cap J_{\beta}=\varnothing$ for all $\beta<\alpha^{*}$. But this contradicts the maximality of $g_{\alpha}$.

Choose an unbounded subset $\Gamma$ of $\omega_{1}$ such that $\alpha<\gamma \in \Gamma$ implies that $\alpha^{*}<\gamma$; let $\Gamma^{*}$ be the set of all limits of $\Gamma$ in $\omega_{1}$. Since $\Gamma^{*}$ is closed and unbounded and $S_{1} \subset S_{0}$, there is an $x \in S_{1} \cap L_{\gamma}$ for some $\gamma \in \Gamma^{*}$. Choose $\gamma_{1}<\gamma_{2}<\ldots$ in $\Gamma$ having $\gamma$ as a limit. By the above paragraph, for each $n \in N$ there is a $\beta_{n}$ such that $x \in J_{\beta_{n}}$ and $J_{\beta_{n}} \in \mathscr{F}_{\gamma_{n}}$. Since $\gamma$ is the limit of $\left\{\delta_{\beta_{n}}\right\},\{x\} \in Z^{*} \subset V$. Also $x$ is a limit point in $S$ of $\left\{y_{\beta_{n}} \mid n \in N\right\}$. So there is an $n$ such that $\left\{y_{\beta_{n}}, x\right\} \in V$. But $\left\{y_{\beta_{n}}, x\right\} \in U$ by the definition of $J_{\beta_{n}}$. This contradicts $U \cap V=\varnothing$.

THEOREM 2. If (A) and (B) hold, then $P_{X} R$ is metric.

Proof. Let $q$ be the set of all subsets of $X$ of the form $\{X\}$ or $\{x \in X \mid p<$ $x\}$ or $\{x \in X \mid x<q\}$ or $\{x \in X \mid p<x<q\}$ for some $p$ and/or $q$ in $X \cap S$. These sets form a basis for the topology of $X$. Since each $K_{\alpha}$ is a Cantor set, for each $\alpha$ there is a countable subset $G_{\alpha}$ of $G$ which is an open basis for $\left(K_{\alpha} \cap X\right)$ in $X$. Let $C_{\alpha}$ be the set of all "end points" ( $p$ 's and $q$ 's in the description above) of members of $\mathscr{G}_{\alpha}$. For each $\alpha \in \omega_{1}$, choose $\alpha^{*} \in \omega_{1}$ so that $C_{\alpha} \subset \operatorname{cl} \cup_{\beta<\alpha^{*}}\left(X \cap K_{\beta}\right)$.

By (A), there is a closed unbounded subset $\Gamma$ of $\omega_{1}$ such that for all $\alpha \in \Gamma$, if $x \in X-\cup_{\beta<\alpha} K_{\beta}$, then $x \notin \operatorname{cl}\left(X \cap\left(\cup_{\beta<\alpha} K_{\beta}\right)\right)$. For each $\alpha \in \Gamma$, let $\Gamma_{\alpha}=\left\{\beta \in \omega_{1} \mid\right.$ if $\alpha<\gamma \in \Gamma$, then $\left.\alpha \leqslant \beta<\gamma\right\}$. We assume that $\Gamma$ was chosen so that $\beta \in \Gamma_{\alpha}$ implies that $\beta^{*} \in \cup_{\gamma \leqslant \alpha} \Gamma_{\gamma}$.

For $\alpha \in \Gamma$, let $X_{\alpha}=\cup_{\beta \in \Gamma_{\alpha}}\left(X \cap K_{\beta}\right)-\cup_{\beta<\alpha} K_{\beta}$. Index $\{I \in$ $\cup_{\beta \in \Gamma_{\alpha}} g_{\beta} \mid I \cap X_{\gamma}=\varnothing$ for $\left.\gamma<\alpha\right\}=\left\{I_{\alpha n} \mid n \in N\right\}$. This is an open basis for $X_{\alpha}$ in $X$. 
If $i \in N$ and $\alpha \in \Gamma$, let $J_{i x}=\cap\left\{I_{\alpha n} \mid n \leqslant i\right.$ and $\left.x \in I_{\alpha n}\right\}$ (one can let $J_{i x}=X$ if $x \notin I_{\alpha n}$ for any $n \leqslant i$ ). For $F \in P_{X} R$ define $U_{i F}=\left\{G \in P_{X} R \mid F\right.$ $\left.\subset G \subset \cup_{x \in F} J_{i x}\right\} ;\left\{U_{i F} \mid i \in N\right\}$ is an open basis for $F$ in $P_{X} R$. For $i \in N$ define

$$
P_{i}\left\{\begin{array}{l|l}
F \in P_{X} R & \begin{array}{l}
\text { If } x \in F \cap X_{\alpha}, \text { then } x \in I_{\alpha n} \text { for some } n<i \\
\text { If } x \in F, z \in F, \text { and } x \neq z \text {, then } J_{i x} \cap J_{i z}=\varnothing
\end{array}
\end{array}\right\} .
$$

By (B), $P_{\left(X \cap K_{\alpha}\right)} R$ is metric and hence, by (D), $X \cap K_{\alpha}$ is countable for all $\alpha \in \omega_{1}$. Thus we can index $X_{\alpha}=\left\{x_{\alpha n} \mid n \in N\right\}$. For $i \in N$, define:

$$
P_{i}^{*}=\left\{F \in P_{i} \mid \text { If } x_{\alpha n} \in F \text { and } x_{\alpha k} \notin F \text { and } k<n \text {, then } x_{\alpha k} \notin \bigcup_{z \in F} J_{i z}\right\} \text {. }
$$

We prove that if $F \in P_{i}^{*}$ and $G \in P_{j}^{*}$ for some $j>i$ and $U_{i F} \cap U_{j G} \neq \varnothing$, then $F \subset G$. Since for any $G \in P_{X} R$ there is a $j>i$ with $G \in P_{j}^{*}$ and there are at most finitely many $F \subset G$, this proves that $\left\{U_{i F} \mid F \in P_{i}^{*}\right\}$ is locally finite for a fixed $i$. The existence of this $\sigma$-locally finite base implies that $P_{X} R$ is metric and proves Theorem 2.

Suppose on the contrary that there is an $H \in U_{i F} \cap U_{j G}$ and an $x \in F-$ $G$. Since $x \in F \subset H \in U_{j G}$, there is a $y \in G$ such that $x \in J_{j y}$. Since $y \in G \subset H \in U_{i F}$, there is a $z \in F$ such that $y \in J_{i z}$. Since $x$ and $z$ belong to $F \in P_{i}, x \notin J_{i z}$ unless $x=z$.

There are $\alpha, \beta$ and $\gamma$ in $\omega_{1}$ such that $z \in X_{\alpha}, y \in X_{\beta}$ and $x \in X_{\gamma}$.

Observe that $\alpha \leqslant \beta \leqslant \gamma$. For suppose $\alpha>\beta$. Since $J_{i z} \subset I_{\alpha n}$ for some $n$ and $I_{\alpha n} \cap X_{\beta}=\varnothing$ for all $\beta<\alpha$, this contradicts $y \in X_{\beta} \cap J_{i z}$. Similarly $\beta \leqslant \gamma$.

Suppose $\alpha<\beta$. Then $\alpha<\gamma$ so $x \neq z$. Since $x \notin J_{i z}$ and $y \in J_{i z}$, there is an end point $p$ of some $I_{\alpha n}$ with $p$ between $x$ and $y$ in $S$; by definition $p \in \operatorname{cl}\left(\cup_{\delta \leqslant \alpha^{*}} X_{\delta}\right)$. Since $\{x, y\} \subset J_{j y}$ and since $J_{j y}$ is an interval, $p \in J_{j y}$. But this is a contradiction since $\alpha^{*}<\beta$ and $J_{j y} \cap\left(\cup_{\delta<\beta} X_{\delta}\right)=\varnothing$.

So we must have $\alpha=\beta$. Recall that $J_{i z}=\bigcap\left\{I_{\alpha n} \mid n \leqslant i\right.$ and $\left.z \in I_{\alpha n}\right\}$. Thus, since $y \in J_{i z} \cap X_{\alpha}$ and $j>i, J_{j y} \subset J_{i z}$. Since $x \in J_{j y}, x \in J_{i z}$. Thus $x=z$.

So $\alpha=\beta=\gamma$ and $x=z$. Since $x=x_{\alpha k}$ and $y=x_{\alpha h}$ for some $h$ and $k$ in $N$ and $x \neq y$, one of $h$ and $k$ is smaller and either $x \notin J_{j y}$ or $y \notin J_{i x}$; but this contradicts $x \in J_{j y}, y \in J_{i z}$, and $x=z$.

THeOREM 3. If (A) and (C) hold then $P_{X} R$ is normal.

Proof. Assuming (A) we define $9, g_{\alpha}, C_{\alpha}, \alpha^{*}, \Gamma, \Gamma_{\alpha}, X_{\alpha}, I_{\alpha n}, J_{i x}, U_{i F}$, and $P_{i}$ exactly as in the proof of Theorem 2.

Now suppose that $Y$ and $Z$ are disjoint closed subsets of $P_{X} R$; we must find disjoint open sets separating $Y$ and $Z$ and thus prove that $P_{X} R$ is normal.

For $F \in P_{X} R$, let $\phi(F)=\left\{\alpha \in \omega_{1} \mid F \cap X_{\alpha} \neq \phi\right\}$. Let $\Delta=\{\langle\phi, J, I\rangle \mid \exists F$ $\in P_{X} R$ such that $\phi=\phi(F)$ and $\left.J=\cup_{x \in F} J_{i x}\right\}$. For $\langle\phi, J, i\rangle \in \Delta$, define 


$$
P_{\langle\phi, J, i\rangle}=\left\{F \in P_{i} \mid \phi=\phi(F), J=\bigcup_{x \in F} J_{i x}\right\} .
$$

Define $Y_{\langle\phi, J, i\rangle}=\left\{F \in P_{\langle\phi, J, i\rangle} \mid Z \cap U_{i F}=\varnothing\right\}$. Then interchanging $Y$ and $Z$ define $Z_{\langle\phi, J, i\rangle}$.

Observe that $Y_{\langle\phi, J, i\rangle}$ and $P_{\langle\phi, J, i\rangle}-Y_{\langle\phi, J, i\rangle}$ are disjoint subsets of $P_{\left(X \cap K_{\text {sup } \phi}\right)} R$ as are $Z_{\langle\phi, J, i\rangle}$ and $P_{\langle\phi, J, i\rangle}-Z_{\langle\phi, J, i\rangle}$. Also all of these sets are closed in $P_{X} R$ since any $F$ belonging to any of them has exactly one member in each of the disjoint $\left\{J_{i x} \mid x \in F\right\}$; and for a fixed $\phi$ and $i$, the possibilities for $\left\{J_{i x} \mid x \in F\right\}$ are finite.

Hence, by (C) there is a function $k_{\langle\phi, J i\rangle}=k: P_{X} R \rightarrow N$ such that $U_{k(F) F} \cap$ $U_{k(G) G}=\varnothing$ whenever $F \in Y_{\langle\phi, J, i\rangle}$ and $G \in P_{\langle\phi, J, i\rangle}-Y_{\langle\phi, J, i\rangle}$ or whenever $G \in Z_{\langle\phi, J, i\rangle}$ and $F \in P_{\langle\phi, J, i\rangle}-Z_{\langle\phi, J, i\rangle}$.

There is also a function $i: P_{X} R \rightarrow N$ such that, if $\phi(F)=\phi, i(F)=i$, and $\cup_{x \in F} J_{i x}=J$, then $F \in Y_{\langle\phi, J, i\rangle}$ if $F \in Y$, and $F \in Z_{\langle\phi, J, i\rangle}$ if $F \in Z$. Observe that $\phi$ and $i$ are finite and that for $\theta \subset \phi$ and $n \leqslant i$ there are only finitely many $K$ with $\langle\theta, K, n\rangle \in \Delta$. So we can also define $j: P_{X} R \rightarrow N$ such that $j(F)>i(F)$ and for all $n \leqslant i(F), \theta \subset \phi(F), G \subset F$, and $\langle\theta, K, n\rangle \in \Delta$, $j(F)>k_{\langle\theta, K, n\rangle}(G)$.

ClaIm. $\cup_{F \in Y} U_{j(F) F}$ and $\cup_{G \in Z} U_{j(G) G}$ are disjoint open sets separating $Y$ and $Z$.

Suppose on the contrary that there are $F \in Y, G \in Z$, and $H \in U_{j(F) F} \cap$ $U_{j(G) G}$. Without loss of generality we assume that $i=i(F) \leqslant i(G)<j(G)=$ $j$.

Since $i<j$, using the proof for Theorem 2, if $x \in F-G$ and $x \in X_{\alpha}$, there is a $y_{x} \in X_{\alpha} \cap G$ such that $x \in J_{j y_{x}}$ and $y \in J_{i x}$. Note that $J_{i x}=J_{i y_{x}}$.

Let $\phi=\phi(F)$ and $J=\cup_{x \in F} J_{i x}$. Then $F \in Y_{\langle\phi, J, i\rangle}$ by the definition of $i=i(F)$.

Let $G^{\prime}=(F \cap G) \cup\left\{y_{x} \mid x \in F-G\right\}$. Clearly $\phi(F)=\phi\left(G^{\prime}\right) \subset \phi(G), J$ $=\cup_{y \in G^{\prime}} J_{i y}$, and $G^{\prime} \subset G \subset H \subset J$. So $G \in U_{i G^{\prime}}$ and $G^{\prime} \in P_{\langle\phi, J, i\rangle}-$ $Y_{\langle\phi, J, i\rangle} \cdot$

Let $H^{\prime}=F \cup G^{\prime}$ and $k=k_{\langle\phi, J, i\rangle}$.

Since $k(F)<j(F)$ we have $F \subset H^{\prime} \subset H \in U_{j(F) F} \subset U_{k(F) F}$ and thus $H^{\prime}$ $\in U_{k(F) F}$.

Since $\phi \subset \phi(G)$ and $i \leqslant i(G), k\left(G^{\prime}\right)<j(G)$. Also $G^{\prime} \subset H^{\prime} \in U_{j(G) G^{\prime}}$ by the definition of $y_{x}$ and $G^{\prime}$. So $H^{\prime} \in U_{k\left(G^{\prime}\right) G^{\prime}}$. But this contradicts $U_{k(F) F} \cap$ $U_{k\left(G^{\prime}\right) G^{\prime}}=\varnothing$ for $F \in Y_{\langle\phi, J, i\rangle}$ and $G^{\prime} \in P_{\langle\phi, J, i\rangle}-Y_{\langle\phi, J, i\rangle}$

THEOREM 4. If $W$ is a subset of a Cantor set $K$, then $P_{W} R$ is normal if and only if $W^{n}$ is a $Q$-set for all $n \in N$.

Proof. Let $K=\{f: N \rightarrow 2\}$.

If $F \in P_{W} R$ and $i \in N$, let $J_{i F}=\{f \uparrow i \mid f \in F\}$ and let $U_{i F}=\{G \in$ $P_{W} R \mid F \subset G$ and $\left.J_{i G} \subset J_{i F}\right\}$. Let $W_{n}=\left\{F \in P_{W} R|| F \mid=n\right\}$ and, for $F \in$ $W_{n}$, let $F^{*} \in W^{n}$ be the natural ordering of $F$ : that is $f<g$ in $F$ if there is a 
$k$ such taht $f(i)=g(i)$ for all $i<k$ but $f(k)<g(k) . P_{W} R$ is normal if for every pair $Y$ and $Z$ of disjoint closed sets there is an $i: P_{W} R \rightarrow N$ such that $U_{i(F) F} \cap U_{i(G) G}=\varnothing$ for all $F \in Y$ and $G \in Z$.

If $S=\left\langle f_{1} \cdots f_{n}\right\rangle \in W^{n}$ and $i \in N$, define $U_{i S}=\left\{\left\langle g_{1} g_{2} \cdots g_{n}\right\rangle \in\right.$ $W^{n} \mid g_{k} \uparrow i=f_{k} \uparrow i$ for all $\left.k \leqslant n\right\}$. Both $Y$ and $W^{n}-Y$ are $G_{\delta}$-sets in $W^{n}$ if and only if there is a function $i: W^{n} \rightarrow N$ such that, if $S \in Y$ and $T \in W^{n}$ - $Y$, then either $S \notin U_{i(T) T}$ or $T \notin U_{i(S) S}$.

If $S=\left\langle f_{1} \cdots f_{n}\right\rangle \in W^{n}$ there is $S^{\prime}=\{f \in S\} \in W_{m}$ for some $m \leqslant n$. Define $W_{m}^{n}=\left\{S \in W^{n} \mid S^{\prime} \in W_{m}\right\}$ and let $t_{S}: n \rightarrow m$ be the unique function such that $f_{j}$ is the $t_{S}(j)$ th term of $\left(S^{\prime}\right)^{*}$. Let $\mathscr{T}_{m}^{n}=\{t: n \rightarrow m\}$ and, for $t \in \mathscr{T}_{m}^{n}$, let $W_{m t}^{n}=\left\{S \in W_{m}^{n} \mid t_{S}=t\right\}$. Observe that each $t \in \mathscr{T}_{m}^{n}$ induces a one-to-one correspondence between $W_{m t}^{n}$ and $W_{m}$ (taking $S$ to $S^{\prime}$ ). Choose $k_{s} \in N$ such that $f \neq g$ in $S$, then $f \uparrow k_{S} \neq g \uparrow k_{s}$. Observe:

$$
\begin{aligned}
& \text { If } S \text { and } T \text { belong to } W_{m}^{n}, i>k_{S} \text {, and } j>k_{T} \text {, then }\left(S^{\prime} \cup T^{\prime}\right) \\
& \in\left(U_{i S^{\prime}} \cap U_{j T^{\prime}}\right) \text { if and only if } T \in U_{i S} \text { and } S \in U_{j T^{*}} \text {. }
\end{aligned}
$$

First we prove that, If $P_{W} R$ is normal and $Y \subset W^{n}$, then $Y$ is a $G_{\delta}$-set in $W^{n}$.

Suppose that $m \leqslant n$ and $t \in \mathscr{T}_{m}^{n}$. It is known that $P_{W} R$ is normal only if it is hereditarily normal. Thus the open subset $\cup_{r \geqslant m} W_{r}$ of $P_{W} R$ is normal. Since $W_{m}$ is closed in $\cup_{r<m} W_{r}$ and discrete in itself, we can find disjoint open sets in $P_{W} R$ separating $Y^{*}$ and $W_{m}-Y^{*}$. Since $t$ induces a one-to-one correspondence between $W_{m}$ and $W_{m v}^{n}$, there is $i: W_{m t}^{n} \rightarrow N$ such that

$$
U_{i(S) S^{\prime}} \cap U_{i(T) T^{\prime}}=\varnothing
$$

if $S \in Y \cap W_{m t}^{n}$ and $T \in W_{m t}^{n}-Y$. Since if $S \in W^{n}, S$ belongs to $W_{m t}^{n}$ for exactly one $m$ and $t, i: W^{n} \rightarrow N$ is well defined; choose $i(S)>k_{S}$ for all $S \in W^{n}$.

This $i$ testifies to $Y$ being a $G_{\delta}$-set for suppose there were an $S=$ $\left\langle f_{1}, \ldots, f_{n}\right\rangle \in\left(Y \cap U_{i(T) T}\right)$ and $T=\left\langle g_{1}, \ldots, g_{n}\right\rangle \in\left(U_{i(S) S}-Y\right)$. Assume without loss of generality that $i(S) \leqslant i(T)$. Since $S \in U_{i(T) T}$ and $k_{S}<i(S)$ $<i(T), f_{j} \uparrow i(T)=g_{j} \uparrow i(T)$ for all $j \leqslant n$. Thus if $S \in W_{m t}^{n}, T \in W_{m t}^{n}$ for the same $m$ and $t$. But this contradicts (*) since $U_{i(S) S^{\prime}} \cap U_{i(T) T^{\prime}}=\varnothing$.

We now prove that, If $W^{n}$ is a $Q$-set for all $n \in N$, then $P_{W} R$ is normal.

Suppose that $Y$ and $Z$ are disjoint closed subsets of $P_{W} R$. For each $m \in N$, let $J_{m}=\{f \uparrow m \mid f \in K\}$ and for $J \subset J_{m}$ let

$$
Y_{J}=\left\{F \in P_{W} R|| J|=| F \mid, J=J_{m F} \text {, and } U_{m F} \cap Z=\varnothing\right\} \text {. }
$$

Let $n=|J|$. Since $\left\{F^{*} \mid F \in Y_{J}\right\}$ and $W^{n}-\left\{F^{*} \mid F \in Y_{J}\right\}$ are both $G_{\delta^{-}}$-sets in $W^{n}$, and, if $G \in\left(W_{n}-Y_{J}\right)$, then $G^{*} \in\left(W^{n}-\left\{F^{*} \mid F \in Y_{J}\right\}\right)$, there is a $j_{J Y}$ or $j: W_{n} \rightarrow N$ such that, if $F \in Y_{J}$ and $G \in W_{n}-Y_{J}$, then either $F^{*} \notin$ $U_{j(G) G^{*}}$ or $G^{*} \notin U_{j(F) F^{*}}$. Thus, by $(*)$,

$$
(F \cup G) \notin U_{j(F) F} \cap U_{j(G) G} .
$$

Interchanging $Y$ and $Z$ define $Z_{J}$ and $j_{J Z}$. 
For each $F \in P_{W} R$ choose $m(F) \in N$ such that for some $J \subset J_{m(F)}$, $F \in Y_{J}$ if $F \in Y, F \in Z_{J}$ if $F \in Z$, and $F \in\left(Y_{J} \cap Z_{J}\right)$ if $F \in P_{W} R-(Y$ $\cup Z$ ). Finally define

$$
\begin{aligned}
i(F)=\operatorname{lub}\left\{j_{J Y}(G)+j_{J Z}(G)\right. & +m(G) \mid G \subset F \\
& \text { and } \left.J \subset J_{m} \text { for some } m \leqslant m(G)\right\} .
\end{aligned}
$$

This $i$ witnesses a separation of $Y$ and $Z$. For suppose there were $F \in Y$, $G \in Z$, and $H \in\left(U_{i(F) F} \cap U_{i(G) G}\right)$. Without loss of generality assume $i(F)$ $\leqslant i(G)$.

Since $F \subset H \in U_{i(G) G}, J_{i(G) F} \subset J_{i(G) G}$. Since $m(F) \leqslant i(F) \leqslant i(G)$ and $|F|=J_{m(F) F}|| F,|=| J_{i(G) F} \mid$ and, for each $f \in F$, there is a $g_{f} \in G$ such that $g_{f} \uparrow i(G)=f \uparrow i(G)$. Let $G^{\prime}=\left\{g_{f} \mid f \in F\right\}$. Then $\left|G^{\prime}\right|=|F|, J_{i(G) F}=J_{i(G) G^{\prime}}$, and $\left(G^{\prime} \cup F\right) \in\left(U_{i(F) F} \cap U_{i(G) G^{\prime}}\right)$. Since $G \subset H \in U_{i(F) F}, J_{i(F) G} \subset J_{i(F) F}$ and thus $G \in U_{i(F) G^{\prime}}$.

Case (1) $m(F) \leqslant m\left(G^{\prime}\right)$. Let $J=J_{m(F)}$. Then, by the definition of $m$, $F \in Y_{J}$. However $G^{\prime} \in\left(W_{|J|}-Y_{J}\right)$ since $m(F) \leqslant i(F)$ and $G \in\left(U_{i(F) G^{\prime}} \cap\right.$ $Z)$. Thus, by the definition of $i, i(F) \geqslant j_{J Y}(F)$ and, since $m(F) \leqslant m\left(G^{\prime}\right)$, $i(G) \geqslant j_{J Y}\left(G^{\prime}\right)$. So, by the definition of $j_{J Y},\left(G^{\prime} \cup F\right) \notin\left(U_{i(F) F} \cap U_{i(G) G^{\prime}}\right)$ which is a contradiction.

Case (2). $m\left(G^{\prime}\right)<m(F)$. Since $m(F) \leqslant i(F), G \in\left(U_{m(F) G^{\prime}} \cap Z\right)$; so $\left(U_{m\left(G^{\prime}\right) G^{\prime}} \cap Z\right) \neq \varnothing$. Thus, by the definition of $m, G^{\prime} \in Z$. Let $J=J_{m\left(G^{\prime}\right) G^{\prime}}$ Then $G^{\prime} \in Z_{J}$ and $F \in\left(W_{|J|}-Z_{J}\right)$. Also $i(G) \geqslant j_{J Z}\left(G^{\prime}\right)$ and, since $m\left(G^{\prime}\right)$ $<m(F), \quad i(F) \geqslant j_{J Z}(F)$. Again $\left(G^{\prime} \cup F\right) \notin\left(U_{i(F) F} \cap U_{i(G) G^{\prime}}\right)$ gives us a contradiction.

\section{BIBLIOGRAPHY}

1. M. E. Rudin, Souslin's conjecture, Amer. Math. Monthly 76 (1969), 1113-1119.

2. C. Pixley and P. Roy, Uncompletable Moore spaces, Proc. Auburn Univ. Topology Conf., 1969.

3. K. Kunen and F. Tall, Between Martin's Axiom and Souslin's Hypothesis, Fund. Math. (to appear).

4. T. Przymusinski and F. Tall, The undecidability of the existence of a nonseparable normal Moore space satisfying the countable chain condition, Fund. Math. 85 (1974), 291-297.

5. H. R. Bennett, W. G. Fleissner and D. J. Lutzer, Metrizability of certain Pixley-Roy spaces (to appear).

Department of Mathematics, University of Wisconsin, Madison, Wisconsin 53706 